\title{
Abaque caractérisant le fonctionnement d'une cheminée déversante à étranglement optimum
}

\section{Design graph for the performance of an overflowing surge tank having optimum throttling conditions}

\author{
pAR L. ESCANDE \\ MEMBRE DE L'INSTITUT \\ INGËNIEUR I.E.T. \\ DIRECTEUR DE L'ÉCOLE NATIONALE SUPÉRIEURE D'S̈LJCTROTECHNIQUE ET D'HYDRALtIOUE DE TOULOUSE
}

\begin{abstract}
On considère un seluil déversant de longueur infinic a la cote $\mathrm{A}$ au-dessus du nivean stalique et une fermeture instantanée.

L'étranglement optimum est défini comme celui qui provoque la mêtme surpression $\mathrm{Y}_{x}$ a l'instant initial d'une part et celui oì le plan d'eau atteint sa cote maximum d'autre part.

On a donc, avec les notations habituelles:

$\mathrm{Y}_{\mathrm{M}}=\mathrm{R}_{0}-\mathrm{P}_{0}=\mathrm{A}$ on $y_{\mathrm{M}}=r_{0}-p_{0}=a$

On pose : $\omega d=\Omega d / F \mathrm{Z}$.

Qa désignant le volume total déversé.

Deux abaques ont été établis donnant en fonction de $p_{a}$ et de a les valeurs de wa et de la surpression muximum en baleur relative $y_{\mathrm{r}}$.
\end{abstract}

Considérons une chambre d'équilibre à étranglement comportant un seuil déversant à une cote $A$ au-dessus du niveau statique et soit $A^{\prime}$ la cote maximum atteinte par le plan d'eau $\left(\mathrm{A}^{\prime}=\mathrm{A}+\mathrm{H}\right)$ au cours du déversement à la suite d'une manœuvre de fermeture instantanée faisant passer le débit des turbines de sa valeur maximum $Q_{0}$ à zéro.

A l'instant initial, le plan d'eau est à la cote $P_{0}$ au-dessous du niveau statique, $P_{0}$ désignant la perte de charge dans le canal d'amenée pour le débit $Q_{0}$, et le débit $Q_{0}$ traverse l'étranglement, en créant une surpression au-dessous de celui-ci, égale à :

$$
\mathbf{Y}_{\mathrm{M}}=\mathbf{R}_{0}-\mathbf{P}_{0}
$$

ou en grandeurs relatives :

$$
y_{m}=r_{0}-p_{0}
$$

Par contre, au moment où le plan d'eau passe par la cote $A^{\prime}$, la vitesse ascensionnelle $V$ s'an-
An infinitely long weir at height A above the static level and an instantaneous shut-down are considered.

The optimum throtle is defined as that which produces the same over-pressure $\mathrm{Y}_{\mathrm{x}}$ at the instant of shut-down as at the instant the water reaches its highest level in the surge tank.

We have therefore, using the usual symbols: $\mathrm{Y}_{\mathrm{M}}=\mathrm{R}_{0}-\mathrm{P}_{0}=\mathrm{A}$ or $y_{\mathrm{m}}=\mathrm{s}_{0}-p_{0}=a$ and we write $\omega d=\left(\Omega d / \mathrm{FZ}^{*}\right)$.

where $\mathrm{Q} d$ is the total volume overflowing. Two graphs have been produced, giving ad and the maximum over-pressure (in terms of $a$ relative value $Y$ ) as a function of $p_{0}$ and $a$.

nule et, avec elle, la perte de charge dans l'étranglement, de telle sorte que la surpression est alors égale à $A^{\prime}$.

Nous définissons l'étranglement optimum comme étant celụi qui provoque la même contrepression à l'instant initial et à l'instant où $Z$ est maximum :

$$
\mathrm{Y}_{\mathrm{M}}=\mathrm{R}_{0}-\mathrm{P}_{0}=\mathrm{A}^{\prime} \quad \text { ou } \quad y_{m}=r_{0}-p_{0}=a^{\prime}
$$

Dans le cas particulier d'un seuil déversant de longueur pratiquement infinie, on a $\mathrm{H}=0$ et $\mathrm{A}^{\prime}=\mathrm{A}$, d'où :

$$
r_{0}=p_{0}+a
$$

Soit $Q_{1}=v_{1} Q_{0}$ le débit qui débouche du canal d'amenée à l'instant $t_{1}$ où le plan d'eau atteint la cote $\mathrm{A}$.

$Q_{1}$ représente le débit déversé maximum, $v_{3}$ étant donné par l'expression (3):

$$
v_{1}^{2}=\frac{1}{2\left(p_{0}+r_{0}\right)^{2}}\left\{1-2\left(p_{0}+r_{0}\right) a-\left[1-2\left(p_{0}+r_{0}\right) r_{0}\right] e^{-2\left(p_{0}+r_{0}\right)\left(p_{0}+a\right)}\right\}
$$


Pratiquement, le maximum de la surpression au-dessous de l'étranglement correspond à l'instant $t_{1}$ et a pour valeur :

$$
\mathrm{Y}_{1}=\mathrm{A}+\mathrm{R}_{3} \quad \text { ou } \quad \mathrm{y}_{1}=a+r_{0} v_{1}^{2}
$$

$Q_{1}$ étant déterminé, le volume déversé total peut être évalué au moyen de la formule :

$$
\Omega_{d}=\frac{\mathrm{W}_{0}{ }^{2}}{2 g} \cdot \frac{\mathrm{L} f}{\mathrm{P}_{10}+\mathrm{R}_{0}} \log \left[1+\frac{\mathrm{P}_{0}+\mathrm{R}_{0}}{\mathrm{~A}} \cdot \frac{\mathrm{Q}_{1}{ }^{2}}{\mathrm{Q}_{0}{ }^{2}}\right]
$$

que nous avons établie $\left(^{\star}\right)$ et dont nous avons eu l'occasion, à diverses reprises, de vérifier le haut degré d'approximation.

On passe aisément aux grandeurs relatives en posant :

$$
\omega_{l l}=\frac{\Omega_{d}}{\mathrm{~F} \mathrm{Z}}
$$

avec :

$$
Z_{*}=\mathrm{W}_{0} \sqrt{\frac{\mathrm{Lf}}{g \mathrm{~F}}}
$$

et les notations habituelles.

On obtient ainsi :

$$
\omega_{d}=\frac{1}{2\left(p_{11}+r_{0}\right)} \log \left[1+\frac{p_{0}+r_{0}}{a} v_{1}^{2}\right]
$$

Nous nous sommes proposés d'établir un abaque donnant, en fonction de $p_{0}$ et de $a$, pour l'étranglement optimum correspondant $r_{0}=p_{0}+a$, les valeurs du volume déversé et de la surpression maximum $y_{1}$, ces diverses quantités étant des grandeurs relatives. Les valeurs de $a$ se trouvent naturellement limitées par la cote maximum $z_{m}$ qu'atteindrait le plan d'eau dans la cheminée, si celle-ci n'était pas déversante. En particulier pour $p_{0}=0$ et $r_{0}=0,707$, étranglement optimum en l'absence de déversement, on a $z_{n}=0,707$ : c'est donc là la plus forte valeur qui puisse être envisagée pour $a$.

La détermination des valeurs limites de $a=z_{m}$, en fonction de $p_{0}$, est immédiate ̀̀ partir des abaques de Calame et Gaden : sur la courbe de la figure $1, a$ est porté en abscisse, $p_{0}$ en ordonnée.

D'autre part, il résulte de l'expression (7) que $\omega_{d}$ devient infini pour $a=0$, e'est-à-dire pour un seuil arasé au niveau statique.

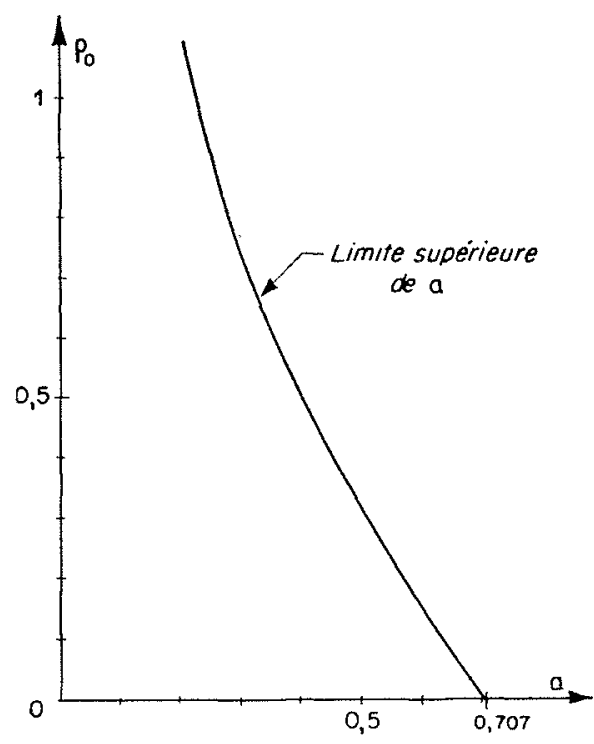

Fic. 1

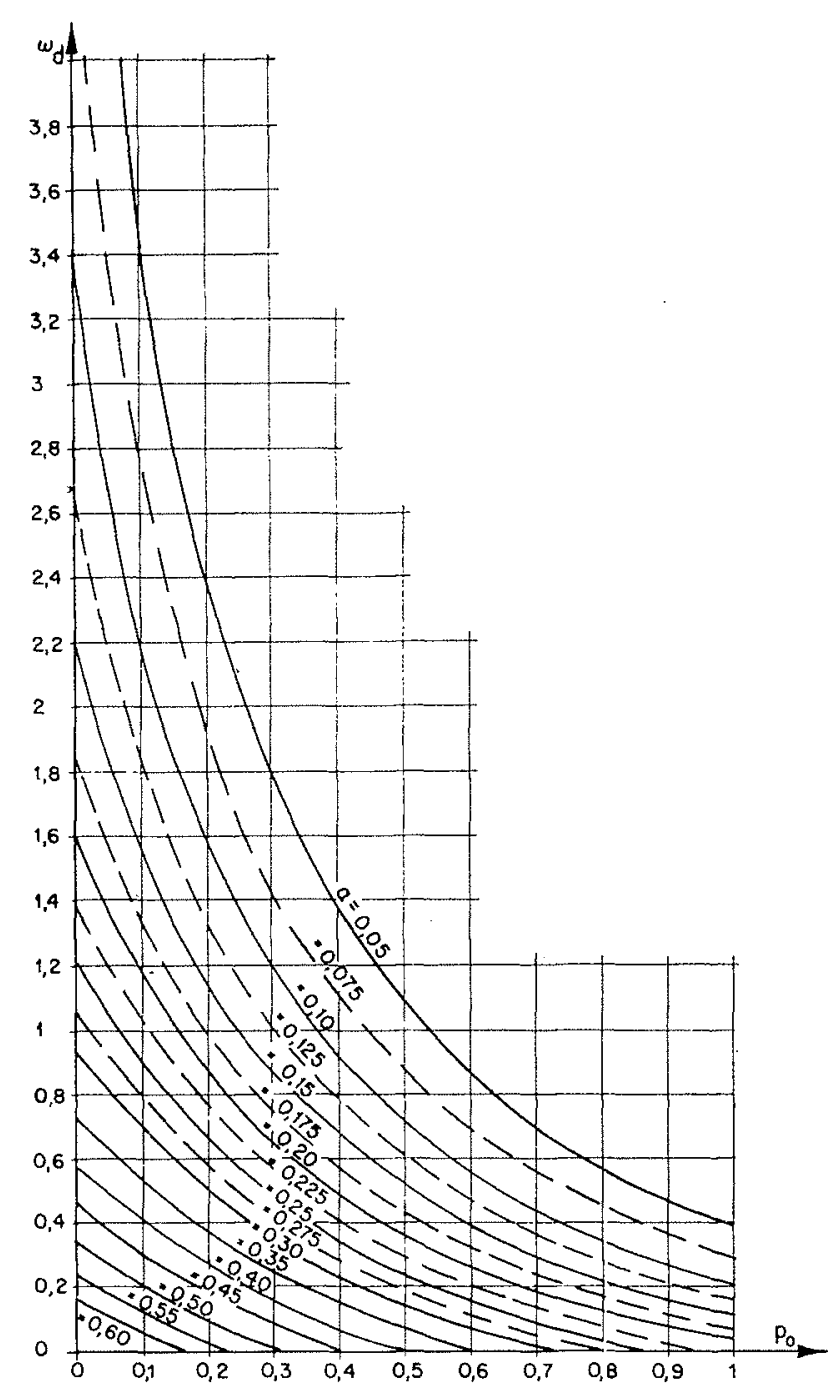

Fig. 2 
Compte tenu de ces éléments, nous avons établi les abaques en faisant varier $p_{0}$ de 0 à 1 et en considérant successivement les valeurs suivantes de $a$ :

$$
0,05-0,1-0,2 \ldots \ldots \ldots-0,7
$$

La figure 2 fournit les valeurs de $\omega_{d}$ et la figure 3 celles de $y_{1}=a+r_{0} v_{1}{ }^{2}$.

Comme on le voit, la surpression maximum peut dépasser notablement les valeurs égales qu'elle possède à l'instant initial, d'une part, et à la fin du déversement, d'autre part.

Il convient donc de tenir compte de cet élément dans le calcul du canal d'amenée et l'abaque de la figure 3 permet de le faire aisément.

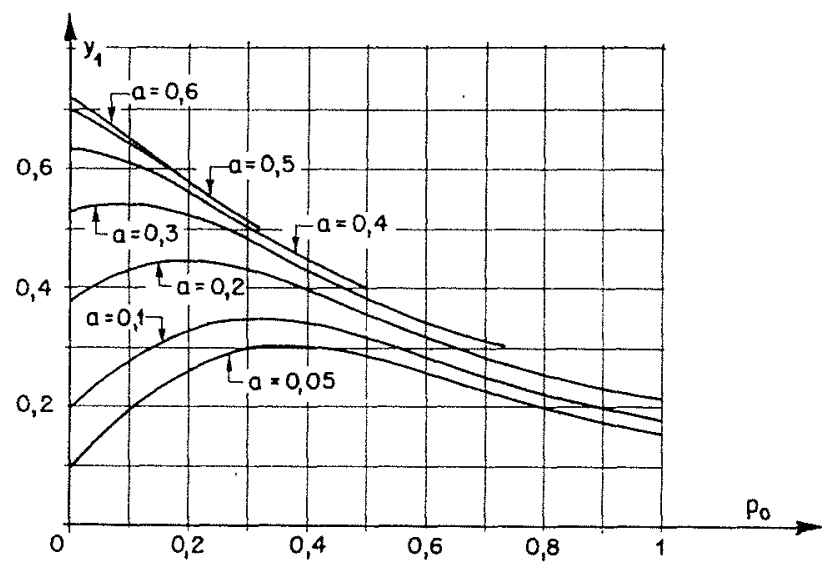

Fig. 3

\section{REMARQUE}

Il nous paraît intéressant de profiter de l'occasion offerte par la présente étude pour répondre à une objection faite parfois aux résultats fournis par l'expression (4) de $\Omega_{d}$, dans le cas où $A=0$ pour lequel le volume déversé devient infini.

Ce résultat n'a rien d'anormal, comme il est lacile de s'en rendre compte par un calcul direct.

L'équation des forces vives s'écril dans ce cas:

$$
\frac{\mathrm{L}}{g} \cdot \frac{d \mathrm{~W}}{d t}+\left(\mathrm{P}_{0}+\mathrm{R}_{n}\right) \mathrm{W}^{2}=0
$$

ou :

$$
d t=--\frac{\mathrm{L}}{g\left(\mathrm{P}_{0}+\mathrm{R}_{0}\right)} \cdot \frac{d \mathrm{~W}}{\mathrm{~W}^{2}}
$$

ou :

$$
t=\frac{\mathrm{L}}{g\left(\mathrm{P}_{0}+\mathrm{R}_{0}\right)} \cdot \frac{1}{\mathrm{~W}}+\mathrm{K}
$$

En tenant compte du fait qu'au début du déversement pris comme instant initial, $W$ est égal à $W_{1}$, on a :

$$
t=\frac{\mathrm{L}}{g\left(\mathrm{P}_{0}+\mathrm{R}_{0}\right)}\left(\frac{1}{\mathrm{~W}}-\frac{1}{\mathrm{~W}_{1}}\right)
$$

el. par suite :

$$
\mathrm{W}=\frac{\mathrm{W}_{1}}{1+\frac{g \mathrm{~W}_{1}\left(\mathrm{P}_{0}+\mathrm{R}_{01}\right)}{\mathrm{L}} t}
$$

Le débit déversant est alors :

$$
\mathrm{Q}_{d}=f \mathrm{~W}=\frac{f \mathrm{~W}_{1}}{1+\frac{g \mathrm{~W}_{1}\left(\mathrm{P}_{0}+\mathrm{R}_{0}\right)}{\mathrm{L}} t}
$$

On voit qu'il tend asymptotiquement vers 0 , quand $t$ devient infini.

Le volume déversé $\Omega_{d}$ est alors donné par l'expression :

$$
\begin{aligned}
\Omega_{d}= & \int_{0}^{\infty} \mathrm{Q} d t=\frac{f \mathrm{~L}}{g\left(\mathrm{P}_{i}+\mathrm{R}_{0}\right)} \\
& \left|\log \left(1+\frac{g W_{1}\left(\mathrm{P}_{0}+\mathrm{R}_{0}\right)}{\mathrm{L}} t\right)\right|_{0}^{\infty}=\infty
\end{aligned}
$$

La puissance dépensée se limite, puisque $A=0$, à celle qui est absorbée par les frottements dus à la perte de charge dans le canal d'amenéc et dans l'étranglement:

$$
\varpi(\mathrm{P}+\mathrm{R}) \mathrm{Q}_{a}=\frac{\bar{\omega}\left(\mathrm{P}_{0}+\mathrm{R}_{0}\right) \cdot f \mathrm{~W}_{1}{ }^{3}}{\left[1+\frac{g \mathrm{~W}_{1}\left(\mathrm{P}_{0}+\mathrm{R}_{0}\right)}{\mathrm{L}} t\right]^{3}}
$$


L'énergie dépensée pendant toute la durée du déversement est donc :

$$
\varpi\left(\mathrm{P}_{0}+\mathrm{R}_{0}\right) / \mathrm{W}_{1}: \times \frac{\mathrm{L}}{g \mathrm{~W}_{1}\left(\mathrm{P}_{0}+\mathrm{R}_{0}\right)} \int_{0}^{\infty} \frac{d\left(1+\frac{g \mathrm{~W}_{1}\left(\mathrm{P}_{0}+\mathrm{R}_{0}\right)}{\mathrm{L}} t\right)}{\left[1+\frac{g \mathrm{~W}_{1}\left(\mathrm{P}_{0}+\mathrm{R}_{0}\right)}{\mathrm{L}} t\right]^{3}}=\varpi--\frac{\mathrm{L} f \mathrm{~W}_{1}{ }^{2}}{2 g}
$$

Or, cette expression représente bien l'énergie cinétique que possède, à l'instant où débute le

déversement, la masse d'eau (๘/g) L. $f$ en mouvement dans le canal d'amenée avec la vitesse $W_{1}$.

\section{DISCUSSION}

Président : M. Жосн

M. le Président remercie à la fois M. Remenieras d'avoir aussi bien présenté la communication et M. Escande d'avoir dégagé dans celle-ci, une fois de plus, et sous un nouvel aspect, la théorie extrêmement complexe des cheminées d'équilibre.

M. Bouvarn se demande si la valeur infiniment grande que prend le volume déversé quand le déversoir est arasé au niveau statique ne provient pas du fait que M. Escande néglige la hauteur de la lame déversante.

En effet l'équation du déversement s'écrit comme suit :

$$
\frac{\mathrm{L}}{g} \frac{d \mathrm{~W}}{d t}=h+(\mathrm{P}+\mathrm{R}) \mathrm{Q}^{2}
$$

$h$ hauteur de la lame déversante,

$P$ coefficient de perte de charge de la galerie,

$R$ coefficient de perte de charge de l'étranglement, le cas échéant.

D'autre part, en cas de fermeture instantanée, le débit déversé $Q$ est de la forme :

et :

$$
\mathrm{Q}=k h^{3 / 2}
$$

$$
\mathrm{W}=\frac{\mathrm{Q}}{f}=\frac{k h^{3 / 2}}{f}
$$

donc le terme $(P+R) Q^{2}$ est de la forme $k h^{3}$ et l'on peut écrire :

$$
\begin{aligned}
\frac{d \mathrm{~W}}{d t} & =\frac{3}{2} \cdot \frac{k}{f} \cdot \sqrt{h} \cdot \frac{d h}{d t}=h+k h^{3} \\
d t & =k \frac{\sqrt{h \cdot d h}}{h+\mathrm{B} h^{3}} \\
t & =k \int \frac{h \cdot d h}{h+\mathrm{B} h^{3}}
\end{aligned}
$$
taire

$$
d \mathrm{~V}=\int \frac{h \cdot d h}{1+\mathrm{B} h^{2}}
$$

et la valeur intégrale :

$$
\mathrm{V}=3 \mathrm{~L} \cdot f \cdot \frac{\mathrm{W}^{2}}{2 g} \cdot \frac{1}{\mathrm{P}} \cdot\left(\log \left(1+\frac{\mathrm{P}}{h_{0}}\right)\right.
$$

ho étant la hauteur initiale de la lame déversante (dans le cas de la cheminée avec étranglement, $P$ est additionné de $R$ ).

On constate donc que $\mathrm{V}$ tend vers l'infini si $\boldsymbol{h}_{0}$ tend vers zéro, mais pratiquement $h_{0}$ n'est jamais nul.
Or, il semble que M. Escande néglige $h$ dans l'écuation (1); il trouve ainsi que $V$ est proportionnel au logarithme du temps, done e'est relativement un petit infini. Si l'on tient compte de ce qu'une certaine énergie est perdue dans le fait qu'il faut monter un peu plus haut, le potentiel est un peu plus grand et il suffit d'ajouter le terme $h$, qui lui-même est très petit, pour donner à ce petit infini une valeur finie. On trouve également que $t$ a une valeur finie, done que le déversement dure un certain temps.

M. Remenieras remarque que le calcul de M. Bouvann est plus approché mais M. Escande a posé, dès le début, que la longueur de la crête déversante était infinie; son calcul est donc correct et logique puisque, malgré son approximation, il $n^{\prime}$ aboutit pas à une énergie infinie.

M. le Président confirme la réponse de M. Remenieras : M. Escande a eu la préoccupation de montrer qu'en fait, l'énergie reste définie.

M. Molbent he croit pas que le sens attribué par M. EsCANDE à l'expression « étranglement optimum 》 corresponde à la définition de cet étranglement dans le cas des cheminées non déversantes : dans ce cas, en effet, l'étranglement optimum est celui qui crée, au début du mouvement, une perte de charge susceptible d'annuler le débit correspondant à la montée maximum; or, pour une cheminée déversante, l'étranglement défini par M. EsCande n'est pas optimum en ce sens que les surpressions de la galerie sont bien supérieures à celle qui provoquent la montée maximum; cet étranglement du fond de la cheminée est donc quelconque et l'intérêt des abaques est beaucoup moins grand parce qu'on ne voit pas pourquoi on choisirait cet étranglement-là plutôt qu'un autre.

M. Rementeras remarque toutefois que M. Escande a explicité, dès le début, ses hypothèses et choisi la définition la plus simple, sinon la plus intéressante en pratique, dc l'ètranglement optimum : il a montré qu'un étranglement ainsi défini n'implique pas qu'au cours de la manœuvre les surpressions ne dépasseront pas légèrement la surpression existant au début de celle-ci Autrement dit, M. Escande s'est efforcé de systématiser le fonctionnement de ce type de cheminées d'équilibre; or, pour réussir cette systématisation, il est nécessaire de choisir des hypothèses de fonctionnement simple.

Pour M. Molbent l'intérêt pratique d'une telle systématisation se trouve réduit du fait que chaque cheminée peut et doit faire l'objet d'une étude particulière.

M. le Président conclut qu'il faut, en cette matière comme dans beaucoup d'autres, savoir ou beaucoup do choses, ou des choses très limitées : il y a intérêt au point de vue pratique, à avoir une représentation graphique simple des phénomènes comme celle que $M$. BERGERON a imaginée pour les coups de bélier et qui couvre de nombreux eas échappant à l'analyse. Or, M. Escande cherche à serrer par des calculs analytiques un grand nombre de cas entre lesquels la détermination se dilue. 